\title{
The Effects of Intravoxel Dephasing and Incomplete Slice Refocusing on Susceptibility Contrast in Gradient-Echo MRI
}

\author{
Jens Frahm, * Klaus-Dietmar Merboldt, and Wolfgang Hänicke \\ Biomedizinische NMR Forschungs GmbH am Max-Planck-Institut für biophysikalische Chemie, Am Faßberg 11, D-37077 Göttingen, Germany
}

Received August 7, 1995

In recent years, the sensitivity of gradient-echo MRI sequences to magnetic field inhomogeneities and tissue susceptibility differences has found an increasing number of applications ranging from an assessment of trabecular bone structure to monitoring intravascular contrast agents or visualizing brain function. In particular, gradient-echo MRI sequences sensitized to alterations of cerebral blood oxygenation (CBO) have resulted in exciting new tools for highresolution mapping of human brain activation. The underlying "functional contrasts" are due to regional and transient changes in the concentration of deoxyhemoglobin. Although many studies have addressed the physiologic origin of MRI susceptibility contrasts and developed models of underlying magnetic field (or frequency) distributions, little attention has been paid to the way in which such effects become modulated by the imaging process itself. As a consequence, the influence of the slice-selection gradient on pertinent gradient-echo images has been largely overlooked.

In a previous study, the signal loss of a gradient-echo image that stems from an additional macroscopic magnetic field inhomogeneity was entirely attributed to a miscalibration of the slice-selection gradient (1). More recently, we have identified the strength of the slice-selection gradient as a factor determining the quality of functional difference maps (2). Here, we further strengthen the analysis of susceptibility contrasts by discussing the influence of all three imaging gradients. We demonstrate that, in general, gradientecho MRI signal changes reflect contributions not only from "'intravoxel dephasing,' but also from incomplete refocusing of the slice-selection gradient. Moreover, while intravoxel dephasing originates from an increase in broadness of the underlying field or frequency distribution, incomplete slice refocusing results from an offset in its mean frequency.

In a first step, we experimentally confirm that a homogeneous shift of the entire frequency distribution results in signal reduction due to incomplete slice refocusing as it is less known than signal loss due to vectorial summation over an ensemble of nuclear spin moments with a broadened

\footnotetext{
* To whom correspondence should be addressed.
}

phase distribution which - at a given echo time-emerges from a corresponding field or frequency distribution. Figures 1 and 2 show gradient-echo images of a water phantom and the brain of a healthy human subject (informed written consent), respectively, that have been acquired in the absence and presence of macroscopic magnetic field gradients. The images were obtained at $2.0 \mathrm{~T}$ (Siemens Magnetom SP4000) with use of the standard imaging head coil, together with spin-density-weighted RF-spoiled FLASH sequences with $T_{2}^{*}$ sensitivity under conditions that are identical to those commonly employed for $\mathrm{CBO}$-sensitive functional brain mapping in this laboratory $(3,4)$.

In the absence of a gradient, Figs. 1a and $2 \mathrm{a}$ exhibit a certain degree of inhomogeneity-induced signal loss that originates from intravoxel dephasing at the relatively long gradient-echo time. The images serve as references for the effects of a gradient along the frequency-encoding axis (Figs. $1 \mathrm{~b}$ and $2 \mathrm{~b}$ ), the slice-selection axis (Figs. 1c and 2c), and the phase-encoding axis (Figs. $1 d-1 f$ and $2 d-2 f$ ). In line with previous investigations $(1,2)$, a further reduction in MRI signal intensity was only seen for the miscalibrated gradient in the slice direction. A gradient in the frequencyencoding axis results in stretching or shrinking of the object along that dimension (depending on relative signs), while its application along the perpendicular phase-encoding direction rotates the effective read gradient to an oblique axis. By comparing respective image raw data sets in Fig. 3, it is seen that inhomogeneity components in the frequency- and phase-encoding direction shift the gradient echo along corresponding directions in $k$ space. Fourier transformation results in altered signal phases, but does not lead to signal loss in magnitude images. Of course, if very strong inhomogeneities push a major portion of the echo outside the acquisition window, the loss of low spatial frequencies results in a complete lack of gross structures and a concomitant edge enhancement (e.g., see Figs. 1e, 1f and 2e, 2f). This phenomenon has already been noted for strongly asymmetric echoes along the frequency- (5) or phase-encoding direction $(6,7)$.

In a second step, we summarize our experimental understanding of inhomogeneity-related signal losses in gradient- 

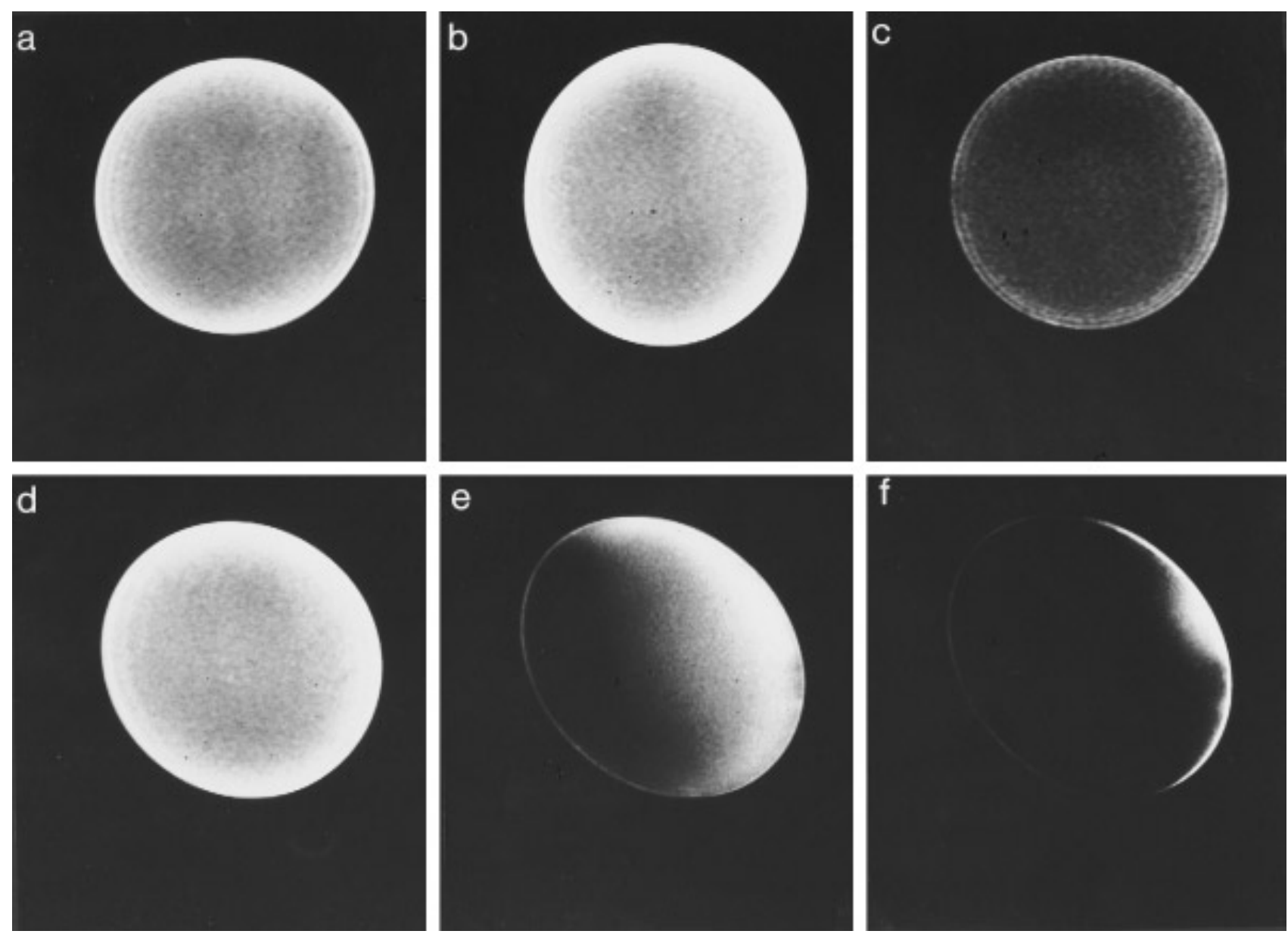

FIG. 1. Susceptibility-sensitized, cross-sectional, gradient-echo images of a water-filled beaker $(2.0 \mathrm{~T}$, RF-spoiled FLASH, TR/TE $=62.5 / 30 \mathrm{ms,}$ $10^{\circ}$ flip angle, measured data matrix $96 \times 256$, field-of-view $150 \times 200 \mathrm{~mm}$, slice thickness $4 \mathrm{~mm}$, measuring time $6.0 \mathrm{~s}$ ). (a) Reference. (b) Application of a $0.1 \mathrm{mT} \mathrm{m}^{-1}$ gradient (corresponding to a $0.75 \mathrm{~A}$ offset of the linear shim current) along the frequency-encoding direction (vertical). (c) Application of a $0.1 \mathrm{mT} \mathrm{m}^{-1}$ gradient along the slice-selection direction. (d-f) Application of a $0.1,0.28$, and $0.32 \mathrm{mT} \mathrm{m}^{-1}$ gradient along the phase-encoding direction (horizontal), respectively.

echo MRI in an integrated perspective. As indicated in Table 1, a magnetic field inhomogeneity may alter the field or frequency distribution of nuclear spin moments in an image voxel in two ways, i.e., by changing its broadness and by shifting its mean value. Although details depend on the actual mechanism, such alterations generally occur in all three spatial dimensions and therefore independently affect the imaging gradients. As far as frequency- and phase-encoding gradients are concerned, intravoxel signal changes are entirely due to a change in broadness of the frequency distribution, while a change in mean frequency shifts the echo position and leads to geometric distortions during image reconstruction. Conversely, however, both types of distribution changes alter the MRI signal intensity when present in the slice-selection direction. While intravoxel dephasing is often even stronger than in the frequency- and phase-encoding direction as section thickness usually surpasses in-plane voxel dimensions, a shift of the entire distribution may easily preclude most spins from refocusing as it causes a net phase offset that further increases with echo time.

Intravascular susceptibility changes in human brain that are associated with activation-related changes in deoxyhemoglobin concentration cause both a change in broadness ( 8 $12)$ and mean $(13,14)$ of the field or frequency distribution of affected spins. Although the relative importance of respective MRI signal changes depends on a large number of unknown variables, the slice-selection process is expected to be particularly sensitive as it benefits from both intravoxel dephasing and incomplete slice refocusing and thus may be exploited to amplify CBO-related signal alterations. For example, these considerations have consequences for optimizing volume coverage in $\mathrm{CBO}$-sensitive functional brain mapping as multislice approaches are likely to be preferable over 3D imaging. This is a consequence of the fact that in 3D gradient-echo MRI both the 2D and 3D phase-encoding gradient may at least partially compensate for magnetic field 

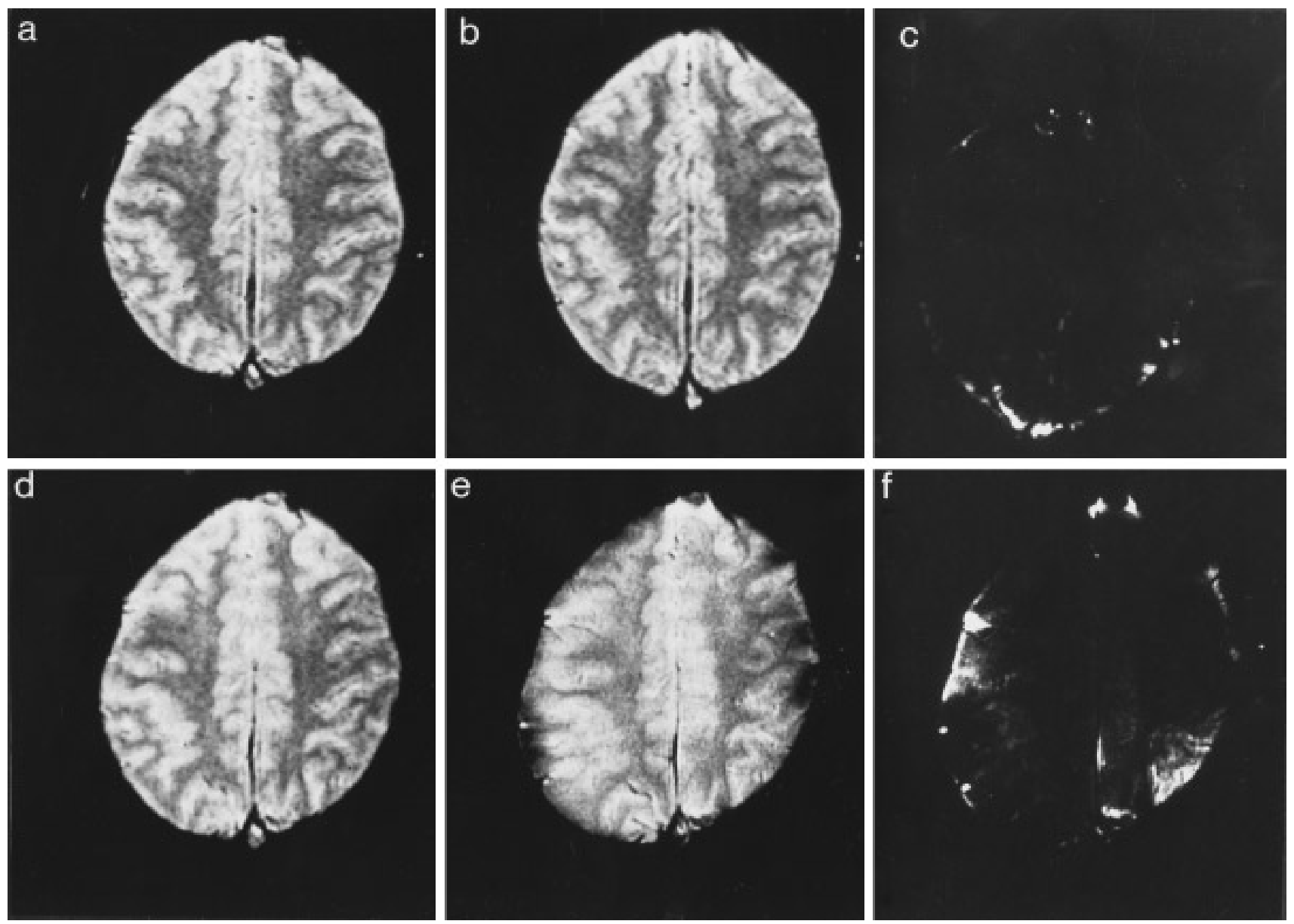

FIG. 2. Susceptibility-sensitized, cross-sectional, gradient-echo images of the brain of a normal volunteer (parameters as described in the legend to Fig. 1). (a) Reference. (b) Application of a $0.1 \mathrm{mT} \mathrm{m}^{-1}$ gradient along the frequency-encoding direction (vertical). (c) Application of a $0.1 \mathrm{mT} \mathrm{m}^{-1}$ gradient along the slice-selection direction. (d-f) Application of a $0.1,0.25$, and $0.28 \mathrm{mT} \mathrm{m}^{-1}$ gradient along the phase-encoding direction (horizontal), respectively.
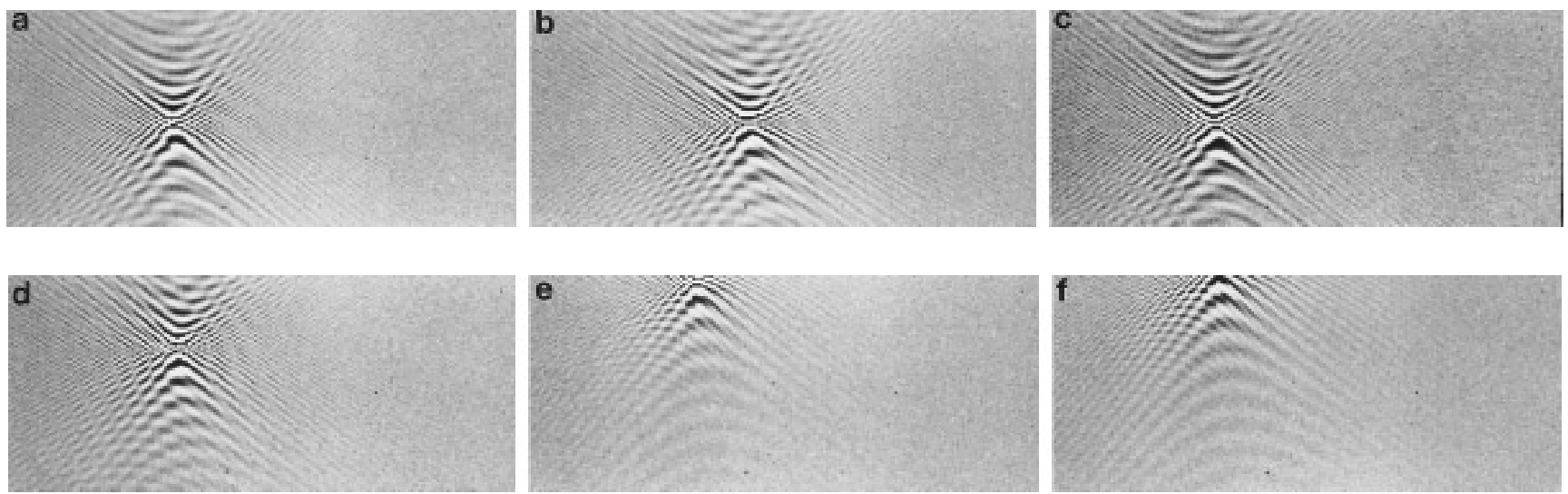

FIG. 3. Raw data (magnitude representation) of the gradient-echo images shown in Fig. 1. (a) Reference (data matrix $96 \times 256$, asymmetric echo). (b) Application of a $0.1 \mathrm{mT} \mathrm{m}^{-1}$ gradient along the frequency-encoding direction (horizontal shift in $k$ space). (c) Application of a $0.1 \mathrm{mT} \mathrm{m}^{-1}$ gradient along the slice-selection direction (no shift but reduced signal-to-noise). (d-f) Application of a $0.1,0.28$, and $0.32 \mathrm{mT} \mathrm{m}^{-1}$ gradient along the phaseencoding direction (vertical shifts in $k$ space), respectively. 
TABLE 1

The Consequences of Magnetic Field Inhomogeneities in a G radient-E cho I mage

Change in field or frequency distribution

\begin{tabular}{cll}
\cline { 2 - 3 } \multicolumn{1}{c}{ MRI gradient } & \multicolumn{1}{c}{ Broadness } & \multicolumn{1}{c}{ Mean } \\
\hline Frequency encoding & $\begin{array}{l}\text { Intravoxel dephasing } \\
(\rightarrow \text { signal loss })\end{array}$ & $\begin{array}{l}\text { Echo shift } \\
(\rightarrow \text { image distortion })\end{array}$ \\
Phase encoding & $\begin{array}{l}\text { Intravoxel dephasing } \\
(\rightarrow \text { signal loss })\end{array}$ & $\begin{array}{l}\text { Echo shift } \\
(\rightarrow \text { image distortion })\end{array}$ \\
Slice selection & $\begin{array}{l}\text { Intravoxel dephasing } \\
(\rightarrow \text { signal loss })\end{array}$ & $\begin{array}{l}\text { Incomplete refocusing } \\
(\rightarrow \text { signal loss })\end{array}$ \\
\hline
\end{tabular}

Note. Changes in broadness or mean of the underlying field or frequency distribution cause either signal loss or geometric distortions, depending on the functionality of the MRI gradients.

inhomogeneities in the manner demonstrated in Figs. 1d, 2d, and $3 \mathrm{~d}$. The more extended the acquisition window along the 3D phase-encoding dimension of $k$ space, the stronger the capacity of the 3D MRI sequence to deal with a locally shifted gradient echo, whereas full sensitivity to magnetic field inhomogeneities is only retained in cross-sectional imaging. On the other hand, these findings again underline that 3D MRI is the method of choice in other applications where unwanted inhomogeneities need to be eliminated as best as possible. Finally, it should be emphasized that the physical relationships discussed here are independent of the actual imaging technique, e.g., FLASH or EPI, as well as of the instrumental, structural, or functional origin of the field inhomogeneity.

\section{REFERENCES}

1. J. Frahm, K. D. Merboldt, and W. Hänicke, Magn. Reson. Med. 6, 474 (1988).

2. J . Frahm, K. D. Merboldt, and W. Hänicke, J. Magn. Reson. B 103, 91 (1994)

3. J. Frahm, K. D. Merboldt, and W. Hänicke, Magn. Reson. Med. 29, 139 (1993).

4. J. Frahm, K. D. Merboldt, W. Hänicke, A. Kleinschmidt, and H. Boecker, NMR Biomed. 7, 45 (1994).

5. G. C. Hurst, J. Hua, O. P. Simonetti, and J. L. Duerk, Magn. Reson. Med. 27, 247 (1992).

6. J. H. den Boef, C. M. van Uijen, and C. D. Holzscherer, Phys. Med. Biol. 29, 857 (1984).

7. P. Margosian and F. Schmitt, Proc. SPIE: Med. Image Process. 593, 6 (1985).

8. C. R. Fisel, J . L. Ackerman, R. B. Buxton, L. Garrido, J . W. Belliveau, B. R. Rosen, and T. J. Brady, Magn. Reson. Med. 17, 336 (1991).

9. S. Ogawa, R. S. Menon, D. W. Tank, S. G. Kim, H. Merkle, J. M. Ellerman, and K. Ugurbil, Biophys. J. 64, 803 (1993).

10. R. P. Kennan, J. Zhong, and J . C. Gore, Magn. Reson. Med. 31, 9 (1994).

11. R. M. Weisskoff, C. S. Zuo, J. L. Boxerman, and B. R. Rosen, Magn. Reson. Med. 31, 601 (1994).

12. D. A. Yablonskiy and E. M. Haacke, Magn. Reson. Med. 32, 749 (1994).

13. A. J esmanowicz, P. A. Bandettini, and J . S. Hyde, Proceedings of the Society of Magnetic Resonance, 2nd Meeting, Berkeley, California, p. 437, 1994.

14. Y. M. Ro and Z. H. Cho, Magn. Reson. Med. 33, 521 (1995). 